\title{
Tuning Silicon-On-Insulator Ring Resonators with In-Plane Switching Liquid Crystals
}

\author{
Wout De Cort ${ }^{1,2, *}$, Jeroen Beeckman ${ }^{2}$, Richard James ${ }^{3}$, F. Anibal Fernandez ${ }^{3}$, \\ Roel Baets ${ }^{1}$, Kristiaan Neyts ${ }^{2}$ \\ ${ }^{1}$ Photonics Research Group \\ Department of Information Technology \\ Ghent University - imec, Gent, Belgium \\ ${ }^{2}$ Liquid Crystals and Photonics Group \\ Department of Electronics and Information Systems \\ Ghent University, Gent, Belgium \\ ${ }^{3}$ Department of Electronics and Electrical Engineering \\ University College London \\ Torrington Place, London, WC1E 7JE, UK \\ *Corresponding author: wout.decort@ugent.be
}

\begin{abstract}
We show that the resonance wavelength of Silicon-On-Insulator ring resonators can be tuned when a top cladding of liquid crystal is present. In-plane strip electrodes are used to generate an electric field that reorients the liquid crystal director in the plane parallel to the chip surface. This causes the resonance wavelength to shift towards longer wavelengths. The magnitude of this shift is about $1 \mathrm{~nm}$, which is twice as large as previously reported shifts. The experimental results are verified extensively with our simulation tools, where a calculation of the director orientation is combined with a fully anisotropic mode solver. From this we get a clear view of the mechanism behind the tuning. (c) 2010 Optical Society of America
\end{abstract}

OCIS codes: $130.7408,160.3710$. 


\section{Introduction}

All-optical telecommunication systems have attracted much scientific and commercial interest as they promise faster and more energy efficient operation than their electrical counterparts. Additionally, they can handle higher bandwidths. There is a growing need for optical components capable of performing efficiently tasks such as filtering wavelengths from a broad spectrum or (de-)multiplexing many optical signals. Silicon-On-Insulator (SOI) is a very popular material system for the fabrication of optical components. There are several reasons for its success. Firstly, it is transparent for wavelengths of $1550 \mathrm{~nm}$ that are used in telecommunication. Secondly, there is a high index contrast between crystalline Silicon $(\mathrm{n}=$ $3.45)$ and its oxide, $\mathrm{SiO}_{2}(\mathrm{n}=1.45)$, that are used to form the waveguides and the insulating layers respectively. This results in a high confinement of light in the waveguides, facilitating the realisation of compact components. Finally, the photonic components can be fabricated using the same techniques used in CMOS fabrication. This mass production leads to a cheap unit cost.

Ring resonators are very interesting optical filters as they typically exhibit sharp resonances for certain wavelengths. In high-index contrast material systems such as SOI these resonators can be made very small with a high Q-factor and low losses [1]. For these reasons they are already used in optical networks. Ring resonators with a tunable resonance are very attractive for reconfigurable optical networks. Possible tuning methods are heating [2] and carrier injection. In SOI these methods lead to a rather limited range of tunability. A third method to achieve tuning is to incorporate appropriate cladding layers on the chip. In these layers a change in refractive index may be induced [3]. The evanescent tail of the light in the waveguide mode extends into the cladding and 'feels' the local change in refractive index. The confinement factor determines the influence of changes in the cladding on the effective index of the mode. As the resonance wavelength of ring resonators is dependent on the effective index it will undergo a similar change due to the cladding layer.

Nematic liquid crystals (LC) are suitable candidates for the cladding layer. They are optically anisotropic materials meaning that the refractive index is directionally dependent. Typical values of $\Delta n$ lie around 0.2 . The director - representing the orientation of the rodshaped molecules - of the LC can be reoriented by an externally applied electric field. When the tuning mechanism is well understood, the large electrooptic effect can be exploited to achieve tuning ranges that can in principle be wider than those achieved with heating or carrier injection. Ring resonators guiding TE-polarized light can be made very small as the modal confinement is high. This enables operation over a large frequency range, but tuning with cladding layers is not trivial as the light does not extend much outside the waveguide. Ring resonators guiding TM-polarized light have been demonstrated previously $[4,5]$. They are more sensitive to changes in the cladding layer at the expense of a smaller free spectral 
range. TE-based ring resonators with LC cladding for tuning have also been demonstrated before although at first the tuning mechanism was not clear [6]. Subsequently it has been shown that, in a configuration where the electric field reorients the director from an orientation parallel to the substrate and the waveguides to an orientation perpendicular to the substrate, the resonances of a ring resonator can be tuned towards shorter wavelengths [7].

In this work we investigate an in-plane switching (IPS) electrode configuration for tuning of the resonance wavelengths. This technique allows for wider tuning ranges than those proposed previously on the SOI platform. We will start with an overview of the device fabrication and then describe briefly the liquid crystal orientation with and without an externally applied electric field. We will then present our experimental results. We demonstrate tuning of the resonance wavelength by in-plane switching of the director. To explain the tuning mechanism we will discuss SOI waveguides with LC cladding and present our simulations, making use of a fully anisotropic modesolver. In this way we can verify our experimental results.

\section{Device Description}

\section{A. Layout and fabrication}

An SOI chip forms the base of our devices (see Fig. 1). It consists of a Si substrate, a 2 $\mu \mathrm{m}$ thick $\mathrm{SiO}_{2}$ layer and a $220 \mathrm{~nm}$ thick monocrystalline $\mathrm{Si}$ layer in which the waveguide structures are defined. The $\mathrm{SiO}_{2}$ layer acts as an optical insulation between the substrate and the waveguides in order to prevent leakage losses. The waveguide dimensions can be very small thanks to the high confinement factor of the material system. The waveguide width is $450 \mathrm{~nm}$ and the height is $220 \mathrm{~nm}$. Bend radii of only a few $\mu \mathrm{m}$ are possible. With UV-curable glue we attach a glass plate on top of the chip. Silica spacers control the spacing which we estimate to be around $5 \mu \mathrm{m}$. The device is then heated on a hotplate together with the LC. In our experiments we use 5CB, 4'-n-pentyl-4- cyanobiphenyl, a commercially available nematic LC. The LC in its isotropic state is deposited near the gap between the chip and the glass. Capillary forces then cause the gap to fill with LC. Finally, the device is cooled gradually to avoid the formation of domains. Prior to assembly the glass plate was spin-coated with an alignment layer. In the experiments discussed here nylon-6 was used to form the alignment layer. After spin-coating and baking, the alignment layer was rubbed with a cloth. When LC comes into contact with the rubbed layer, the director will orient itself along the rubbing direction. In this way we can control the initial orientation of the director. The finger electrodes (see Fig. 2) are made by photolithography of the very thin Indium Tin Oxide (ITO) layer that coats the glass. The fingers are typically a few $\mathrm{mm}$ long, around $10 \mu \mathrm{m}$ wide and spaced by $10-20 \mu \mathrm{m}$. They end in large contact pads with metallic wires attached to ease electrical connection. 


\section{B. LC orientation}

On the glass plate the rubbing provides a fixed director orientation. On the surface of the SOI chip an alignment layer would weaken the effect of the LC on the waveguide mode due to its thickness and the anchoring forces that inhibit reorientation of the surface director. Maximal tuning will be achieved with very weak adhesion of the molecules to the surface. This is, in practice, hard to achieve as the anchoring energy always has a finite value [8] and the molecules experience some anchoring forces even when no alignment layer is present. The anchoring strength of the rubbed nylon- 6 surface is very high. It is important to understand what exactly happens on the surface of the chip before and during the tuning. Experiments with a polarizating microscope can provide valuable insight into this.

We take a close look at one of our ring resonators clad with a top layer of LC. The orientation of the director at the glass plate is fixed by the nylon alignment layer, parallel to the waveguides as determined by the rubbing direction. On the chip surface there is no alignment layer. However, from experience we know that the director is degenerate on the plane parallel to the $\mathrm{Si}$ or $\mathrm{SiO}_{2}$ substrates. However, elastic forces stemming from the upper alignment cause alignment parallel to the waveguides at the $\mathrm{Si}$ or $\mathrm{SiO}_{2}$ surfaces. The polarizer and analyzer of the microscope are set perpendicular to each other and the polarizer is parallel to the rubbing direction. We see in the left hand side of Fig. 3 that most of the microscope image is dark. Upon incidence, only the mode along the extraordinary axis of the LC is excited and the polarization is not changed throughout the device. We can conclude that the director remains parallel to the rubbing direction at the top glass plate. We also see that the image is bright at the left and right side of the ring. On a structured surface like our chip, the LC director will orient itself in such a manner that the energy is at a minimum [9-12]. The director will strive for an orientation parallel to the surface but also to the vertical sidewalls. The bright regions in Fig. 3 therefore indicate a twist of the director at the side of the trench around the ring and to the sidewall of the ring waveguide itself. This results in a rotation of the polarization of the light and gives rise to bright parts of the image. A schematic view of the orientation is shown in the right part of Fig. 3. We can conclude that on the chip surface the director retains the orientation forced by the rubbing layer except near the etched structures where it follows the ribs of intersecting surfaces [11]. In the close vicinity of the waveguide the director is oriented parallel to the propagation direction of the light. When the director orientation on the chip is perpendicular to the rubbing direction the director can turn either clockwise or counterclockwise. These situations are energetically equivalent and when they exist next to each other they are separated by a disclination line. The dark interruptions on both sides of the ring are examples of this. The situation completely changes when an electric field is present. In bulk LC the director will reorient along the field lines as soon as the field is strong enough to overcome the elastic forces holding the LC. This 
transition at a certain threshold field strength is called the Freedericksz transition. At the surface of the chip the effect will not be as strong as in the bulk because the molecules are held to some extent by the surface anchoring forces. It is not known how strong these forces exactly are. We defined lithographically ITO finger electrodes on the glass plate. Applying a voltage results in an electric field with field lines perpendicular to the rubbing direction and parallel to the substrate surface. We can see the effect of this under the polarization microscope (the left part of Fig. 4). Note the difference between the region near the electrodes where the dominant field is between the SOI chip and the finger and the region between the fingers where the director is twisted in the plane parallel to the surface. Below the fingers the director reorients vertically and the image is dark. Between the fingers everything is bright indicating a twist of the director. A schematic view of the orientation with a certain voltage applied is given in the right part of Fig. 4. To see what the influence of this change in director orientation is on the waveguide mode we have to inspect the output of the component.

\section{Experimental results}

\section{A. Measurement details}

For ease of alignment and quick measurements we equip our waveguides with grating couplers. The $70 \mathrm{~nm}$ deep etched teeth of these periodical structures couple light from an optical fiber to the nanophotonic waveguides and vice versa [13]. The fiber shines the light vertically on the grating couplers and about $30 \%$ is transferred into the waveguide mode. As a light source we can use a tunable laser or a broadband source like a superluminescent LED. Polarization controllers ensure that TE polarized light is coupled in. The second grating coupler sends the light again to an optical fiber where it is fed to either a power detector or a spectrum analyzer, depending on the light source used. To apply the electric field we use a signal generator. The signal is a square wave of typically $10 \mathrm{kHz}$ frequency. The AC signal prevents ion drift between the electrodes. We can now measure the output at different wavelengths for different values of the voltage between the fingers of the electrodes.

\section{B. Tuning the resonance}

The ring has a bend radius of $4 \mu \mathrm{m}$ and 4 straight sections of $1 \mu \mathrm{m}$. The output spectrum, for different values of the voltage is shown in Fig. 5. We see a clear shift towards longer wavelengths for increasing voltages. The magnitude of the shift is about $1 \mathrm{~nm}$ which is the largest reported tuning range for TE-based devices tuned with al LC cladding. While the resonance dips are still well pronounced, they are not as deep as when the rings are clad with air. The LC introduces some scattering losses which reduces the performance of the rings. The resulting Q-factors are around 5000 for the untuned ring and drop to around 3500 for the fully tuned ring. This corresponds with propagation losses of $34 \mathrm{~dB} / \mathrm{cm}$ for an untuned ring 
and $36 \mathrm{~dB} / \mathrm{cm}$ for a tuned ring. These rather high loss values can be attributed mostly to scattering of the light by the LC. A good uniformity of the LC on the chip surface can reduce these values. However, such a good uniformity is often hard to achieve without additional alignment layers on the surface. Adding an alignment layer will reduce the interaction of the light with the LC and the resulting tuning will be weaker. Therefore we have chosen to leave the surface of the chip uncoated. When we track the position of the dip as a function of voltage we obtain the graph Fig. 6. We see that there is a threshold value for the electric field to overcome the elastic forces holding the LC. After that a small blue shift in the resonance wavelength occurs. The resonance wavelength then shifts to longer wavelengths until it saturates for high voltages. This value occurs when all molecules have reoriented to their maximum twist angle. A further increase in voltage does not result in an additional shift. Currently, we do not have a clear explanation for the small blue shift observed. It could be due to a vertical reorientation of the director occurring at low voltages when the field between the fingers and substrate is dominant.

\section{Tuning mechanism explained}

\section{A. Waveguides with LC cladding}

The effective index of a waveguide is determined by the interaction of the light with the materials in which it propagates. As the mode in our SOI waveguides has evanescent tails extending into the cladding layers both the oxide and the LC top cladding will contribute to the effective index. The refractive index of the $\mathrm{LC} n_{L C}=\sqrt{\epsilon_{r L C}}$ is determined by the interaction of the electric field components of the light with the relative dielectric constants of the LC. While our devices are designed for TE-polarized light, the mode has nonzero yand z-components due to the small dimensions. The transverse $\mathrm{x}$-component of the electric field is the strongest component in the $\mathrm{Si}$, but near the sidewalls of the waveguide the longitudinal z-component is very strong. The y-component is generally very small and we will not take it into account here [7]. In the cells the LC director has an orientation parallel to the propagation direction of the light in the absence of an electric field. When we apply a voltage between the fingers of the electrodes the resulting field will reorient the director in the plane parallel to the chip. It is readily seen that in the initial orientation the $\mathrm{x}$ component of the electric field experiences a low dielectric constant as the molecules present their short axis. The z-component 'sees' the long axis of the molecules and a high value of the dielectric constant. When the director turns the situation changes. The z-component experiences a reduction in dielectric constant whereas the $\mathrm{x}$-component now sees a higher value. The two situations are depicted in Fig. 7. We expect that the x-component will have the strongest influence on the effective index as it is strongly pronounced at the sides and the top of the waveguide. The longitudinal component is only situated at the sidewalls. These 
considerations indicate that the effective index will increase when a voltage is applied and we expect the resonance wavelength of ring resonators to shift to higher wavelengths like we have seen experimentally. To verify whether our assumptions are correct we calculate numerically the effective index of SOI waveguides with LC cladding.

\section{B. Simulations}

We use $5 \mathrm{CB}$ as LC which is a common nematic LC with uniaxial properties. When solving the modes of a waveguide with this material on top the dielectric tensor can contain in principle 9 non-zero elements. This makes the problem difficult to solve. When longitudinal anisotropy is incorporated there is an asymmetry with respect to the z-direction. This leads to off-axis energy transfer of the extraordinary polarization component [14-16]. We have developed a mode solver capable of handling fully anisotropic problems. The calculation is based on the solution of the variational form of the curl-curl equation of the electric field, implemented with higher-order edge elements [17]. First we want to check the relative importance of the $\mathrm{x}$ - and the z-components on the effective index of the mode. We compare the cases where the refractive index is high in the $\mathrm{x}_{-}, \mathrm{y}-$, or $\mathrm{z}$-direction. The values of the effective index can be seen in Fig. 8. We use values for the cladding layer that are approximately those of 5CB, $n_{e}=1.71$ along the long (extra-ordinary) axis and $n_{o}=1.53$ along the short (ordinary) axis. The waveguide under investigation is $450 \mathrm{~nm}$ wide and $220 \mathrm{~nm}$ high. The values for the effective indices can be seen in Fig 8. We also include the values for isotropic cladding with the ordinary and extra-ordinary refractive indices.

We see that a change in the dielectric constant along the $\mathrm{x}$ axis has the strongest effect on the effective index. Therefore, we expect a larger tuning range for in-plane switching devices compared to the devices discussed in [7], which is confirmed by the experiments. If the anchoring on the surface were zero and the voltage high enough we could in principle reach the situation shown in Fig. 7 and $\Delta n_{\text {eff }}$ would be 0.016 . This is however not a realistic situation as the anchoring on a real chip will have a non-zero value which will not allow the director to rotate freely. The numerical calculation of the director orientation in this geometry and with an electric field present is difficult as several equations have to be solved at once. We developed a tool to solve this problem. A variable order approach [18] is taken based on the minimization of the Landau-de Gennes free energy functional [9]. We implement this model in a finite-element scheme. As the degree of order of the liquid crystal can vary it is possible to model defects where there is a rapid variation in the order parameter. With this tool we can model optical waveguides in combination with LC. The permittivity distribution that results from the liquid crystal modelling is taken into account in the modal solver when calculating the optical fields.

The outline of the simulation space can be seen in Fig. 9. A $1 \mu$ m thick $\mathrm{SiO}_{2}$ layer supports 
the waveguide which is $450 \mathrm{~nm}$ wide and $220 \mathrm{~nm}$ high. The LC is initially oriented parallel to the waveguide, with a small pretwist of 2 degrees. Above the LC we have defined a thin layer of glass where 2 electrodes are defined. For sufficiently high voltages the field rotates the LC director. For different voltages we calculate the director orientation (Fig. 9) and the effective index of the mode. A horizontal cut through the mode profiles is presented in Fig. 10. We can translate the change in effective index to a change in resonance wavelength of ring resonators with the following equation:

$$
\Delta n_{e f f} / n_{g}=\Delta \lambda / \lambda
$$

where $n_{g}$ is the group index. We find a curve in Fig. 11 very similar to what we found in the experiments described earlier. Simulations were performed for different values of the anchoring at the Si surface. Strong anchoring allows no twist or tilt of the director on the surface, but deviations become possible for finite values of the anchoring strength. We can clearly distinguish the threshold voltage and the saturation behaviour at higher voltages. The fact that the threshold value in the experiments is found to be higher is because the spacing of the electrodes is smaller in the simulations. We choose the smaller spacing to reduce the complexity and the calculation time. It is also easily understood why the tuning range is bigger than in the experiments. As we work with circular structures, only part of the ring experiences the maximal director reorientation while other parts see no change in orientation at all. It is however important that we have determined and verified the mechanism behind the tuning of ring resonators with LC cladding. It can be seen from the simulation results that there certainly is still room for improvement as the maximal tuning range can be around $6 \mathrm{~nm}$. This will require electrodes specifically designed for ring resonators as every part of the ring needs to be tuned in an optimal fashion.

\section{Conclusions}

We have fabricated LC cells consisting of an SOI chip equipped with ring resonators, a layer of LC and a glass plate with an alignment layer. ITO finger electrodes are defined on the glass plate. When a voltage is applied the electric field reorients the LC in the plane parallel to the chip surface. This shifts the resonance wavelength of the rings about $1 \mathrm{~nm}$ towards longer wavelengths. We have shown that the electric field components of the light extend and interact with the LC cladding. The net result is an increase in effective index when using the proposed IPS configuration. The waveguide modes were simulated using a combination of two tools. Firstly, we calculate the orientation of the liquid crystal in the presence of an electric field. The permittivity tensor that results is then imported in our fully anisotropic

mode solver. The outcome of the simulations confirms the suggested tuning mechanism and explains the tuning curves found in experiment. 


\section{Acknowledgements}

W. De Cort acknowledges the Institute for the Promotion of Innovation through Science and Technology in Flanders (IWTVlaanderen) for a specialization grant. Jeroen Beeckman is Postdoctoral Fellow of the Research Foundation Flanders (FWO-Vlaanderen). The work has been carried out in the framework of the IAP project Photonics@be of the Belgian Science Policy.

\section{References}

[1] P. Dumon, W. Bogaerts, V. Wiaux, J. Wouters, S. Beckx, J. Van Campenhout, D. Taillaert, B. Luysaert, P. Bienstman, D. Van Thourhout and R. Baets, "Low-Loss SOI Photonic Wires and Ring Resonators Fabricated With Deep UV Lithography," IEEE Photonics Technol. Lett. 16, 1328-1330 (2004)

[2] F. Gan, T. Barwicz, M. A. Popovic, M. S. Dahlem, C.W. Holzwarth, P. T. Rakich, H. I. Smith, E.P. Ippen and F.X. Kartner, "Maximizing the Thermo-Optic Tuning Range of Silicon Photonic Structures," in Proceedings of 2007 Photonics in Switching, San Francisco, CA, USA (2007).

[3] G.H. Haertlin and C. E. Land, "Hot-pressed (PB,LA)(ZR,TI)O3 Ferroelectric Ceramics for Electrooptic Applications," J. Am. Ceram. Soc. 54, 1-11 (1971)

[4] A. Di Falco and G. Assanto, "Tunable wavelength-selective add-drop in liquid crystals on a silicon microresonator," Opt. Commun. 279, 210-213 (2007)

[5] V.G. Chigrinov, L. Zhou, A.A. Muravsky, A.W.O. Poon, "Electrically tunable microresonators using photoaligned liquid crystals," US Patent Application Publication, US 2007/0258677 A1, Nov. 8, 2007

[6] B. Maune, R. Lawson, C. Gunn, A. Scherer and L. Dalton, "Electrically tunable ring resonators incorporating nematic liquid crystals as cladding layers," Appl. Phys. Lett. 83, 4689-4691 (2003)

[7] W. De Cort, J. Beeckman, R. James, F. A. Fernandez, R. Baets and K. Neyts, "Tuning of silicon-on-insulator ring resonators with liquid crystal cladding using the longitudinal field component," Opt. Lett. 34, 2054-2056 (2009)

[8] C. Desimpel, J. Beeckman, K. Neyts, S. Verstuyft, D. Van Thourhout, K. d'Havé and P. Rudquist, "Realization of a Four-Electrode Liquid Crystal Device With Full In-Plane Director Rotation," IEEE T. Electron. Dev. 54, 1295-1300 (2007)

[9] P. G. de Gennes and J. Prost, The Physics of Liquid Crystals (Oxford: Oxford University Press, 1995)

[10] J. C. Jones, G. Bryan-Brown, E. Wood, A. Graham, P. Brett and J. Hughes, "Novel bistable liquid crystal displays based on grating alignment," in Proceedings of the Society 
of Photo-Optical Instrumentation Engineers (SPIE), San José, CA, USA, 84-93 (2000)

[11] H. Desmet, K. Neyts, R. Baets, "Modeling nematic liquid crystals in the neigborhood of edges," J. Appl. Phys 98, 123517 (2005)

[12] B. Bellini and R. Beccherelli, "Modelling, design and analysis of liquid crystal waveguides in preferentially etched silicon grooves," J. Phys. D Appl. Phys. 42, 045111 (2009)

[13] D. Taillaert, P. Bienstman and R. Baets, "Compact efficient broadband grating coupler for silicon-on-insulator waveguides," Opt. Lett. 29, 2749-2751 (2004)

[14] E. Gros and L. Dupont, "Beam deflector using double-refraction in ferroelectric liquid crystal waveguides," Ferroelectrics 246, 1125-1132 (2000)

[15] J. Beeckman, K. Neyts, X. Hutsebaut, C. Cambournac and M. Haelterman, "Simulation of 2-d lateral light propagation in nematic-liquid-crystal cells with tilted molecules and nonlinear reorientational effect," Opt. Quant. Electron. 37, 95-106 (2005)

[16] J. Beeckman, K. Neyts, X. Hutsebaut and M. Haelterman, "Observation of out-coupling of a nematicon," Opto-Electron. Rev. 14, 263-267 (2006)

[17] J. Beeckman, R. James, F. A. Fernández, W. De Cort, P. J. M. Vanbrabant and K. Neyts, "Calculation of Fully Anisotropic Liquid Crystal Waveguide Modes," J. Lightwave Technol. 27, 3812-3819 (2009)

[18] R. James, E. Willman, F. A. Fernandez and S. E. Day, "Finite-element modeling of liquid crystal hydrodynamics with a variable degree of order," IEEE T. Electron. Dev. 53, 1575-1582 (2006) 


\section{List of Figure Captions}

Fig. 1. Schematic view of a device consisting of a SOI substrate with LC cladding, sealed off by a glassplate with finger electrodes and alignment layer.

Fig. 2. Schematic top view of the finger electrode structure. On the right side a schematic view of a ring resonator between two interdigital electrodes is shown.

Fig. 3. Left: Image of a ring resonator with LC cladding, no voltage applied. The straight waveguide is indicated in grey. Right: Schematic view of the director orientation over the structure. The orientation of the polarizer $(\mathrm{P})$, analyzer $(\mathrm{A})$ and rubbing $(\mathrm{R})$ are indicated in the top left corner.

Fig. 4. Left: Image of a ring resonator with LC cladding, with $80 \mathrm{~V}$ between the fingers. The straight waveguide is indicated in grey. The region between the fingers (which remain dark) becomes bright as the molecules twist. Right: Schematic view of the director orientation over the structure. The orientation of the polarizer $(\mathrm{P})$, analyzer $(\mathrm{A})$ and rubbing $(\mathrm{R})$ are indicated in the top left corner.

Fig. 5. The resonance dips shift towards longer wavelengths for increasing voltages.

Fig. 6. When we trace the resonance wavelength for increasing voltages we find a curve with a threshold value and a saturation effect for high voltages.

Fig. 7. When there is no voltage applied, the molecules align parallel to the waveguide. With the electric field present the molecules reorient in the plane parallel to the surface to align along the field lines perpendicular to the waveguide.

Fig. 8. We compare the effective index of the waveguide mode for different LC orientations. From left to right: $\mathrm{LC}$ oriented along $\mathrm{x}^{-}, \mathrm{y}-$, and $\mathrm{z}$-axis, uniform refractive index equal to the ordinary refractive index of the LC and uniform refractive index equal to the extra-ordinary refractive index of the LC.

Fig. 9. We simulate the director orientation of the LC cladding on top of the waveguide in the presence of an electric field. The results are imported in the mode solver.

Fig. 10. With our mode solver we can calculate the $\mathrm{x}-, \mathrm{y}$ - and z-polarized fields of the light. We present here the normalized fields along a horizontal cut through the waveguide (see dotted line in Fig. 1).

Fig. 11. Simulated resonance wavelength for increasing voltages. Three different values for the anchoring are used. For lower values of the anchoring on the surface the tuning range is wider and the saturation appears at lower voltages. 


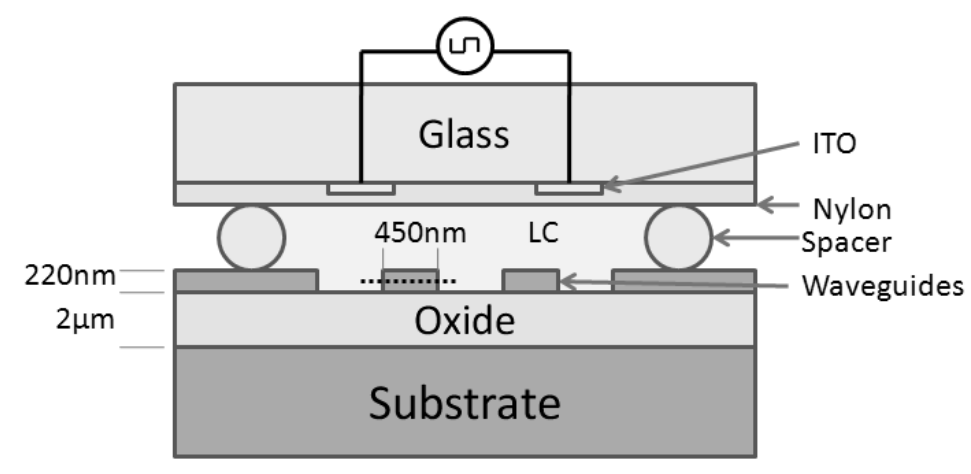

Fig. 1. Schematic view of a device consisting of a SOI substrate with LC cladding, sealed off by a glassplate with finger electrodes and alignment layer. 


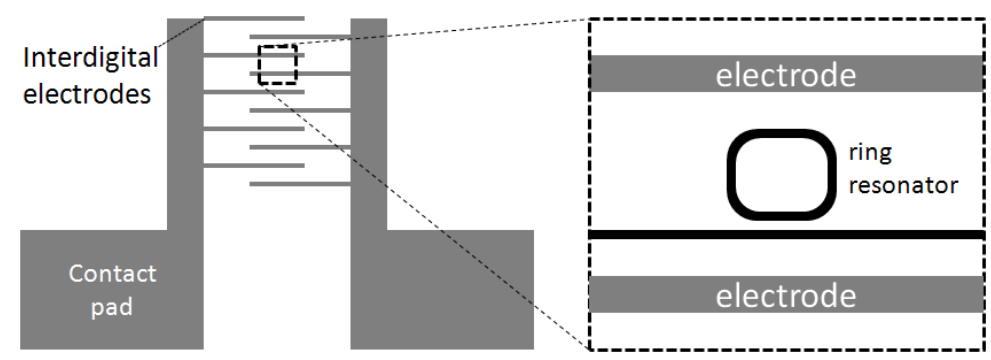

Fig. 2. Schematic top view of the finger electrode structure. On the right side a schematic view of a ring resonator between two interdigital electrodes is shown. 

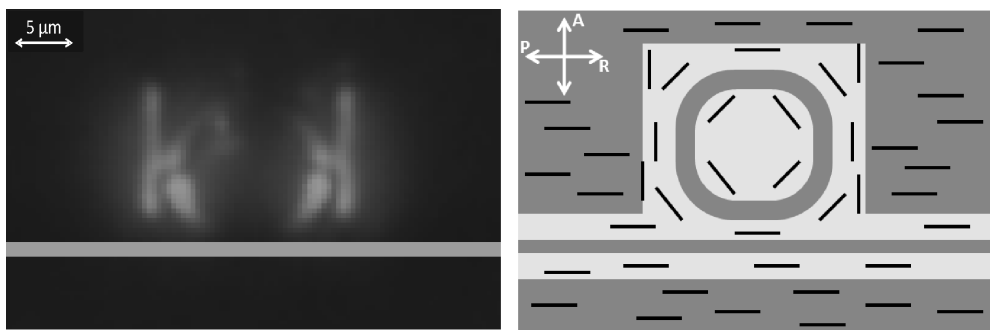

Fig. 3. Left: Image of a ring resonator with LC cladding, no voltage applied. The straight waveguide is indicated in grey. Right: Schematic view of the director orientation over the structure. The orientation of the polarizer $(\mathrm{P})$, analyzer (A) and rubbing $(\mathrm{R})$ are indicated in the top left corner. 

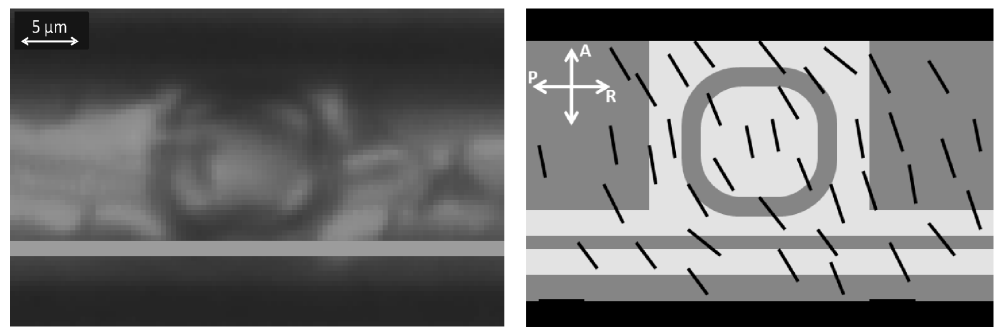

Fig. 4. Left: Image of a ring resonator with LC cladding, with $80 \mathrm{~V}$ between the fingers. The straight waveguide is indicated in grey. The region between the fingers (which remain dark) becomes bright as the molecules twist. Right: Schematic view of the director orientation over the structure. The orientation of the polarizer $(\mathrm{P})$, analyzer $(\mathrm{A})$ and rubbing $(\mathrm{R})$ are indicated in the top left corner. 


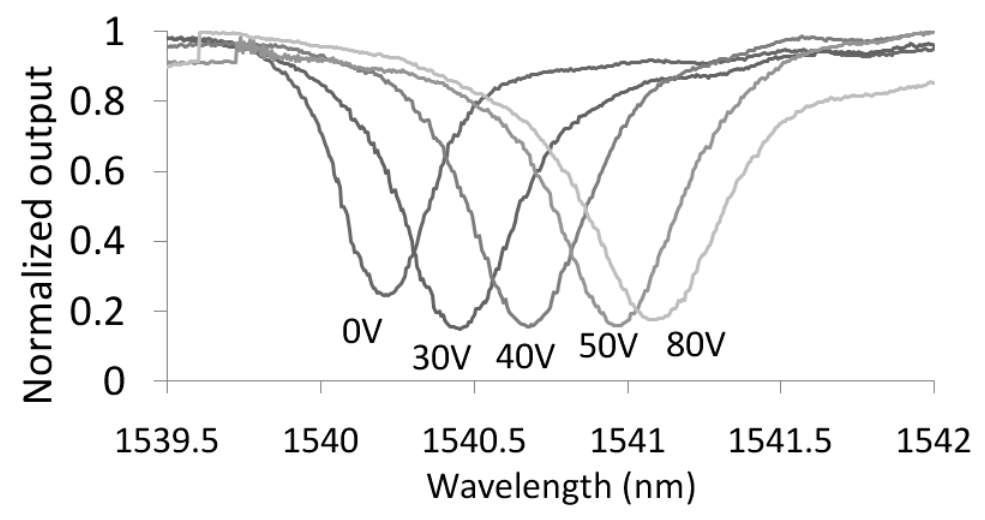

Fig. 5. The resonance dips shift towards longer wavelengths for increasing voltages. 


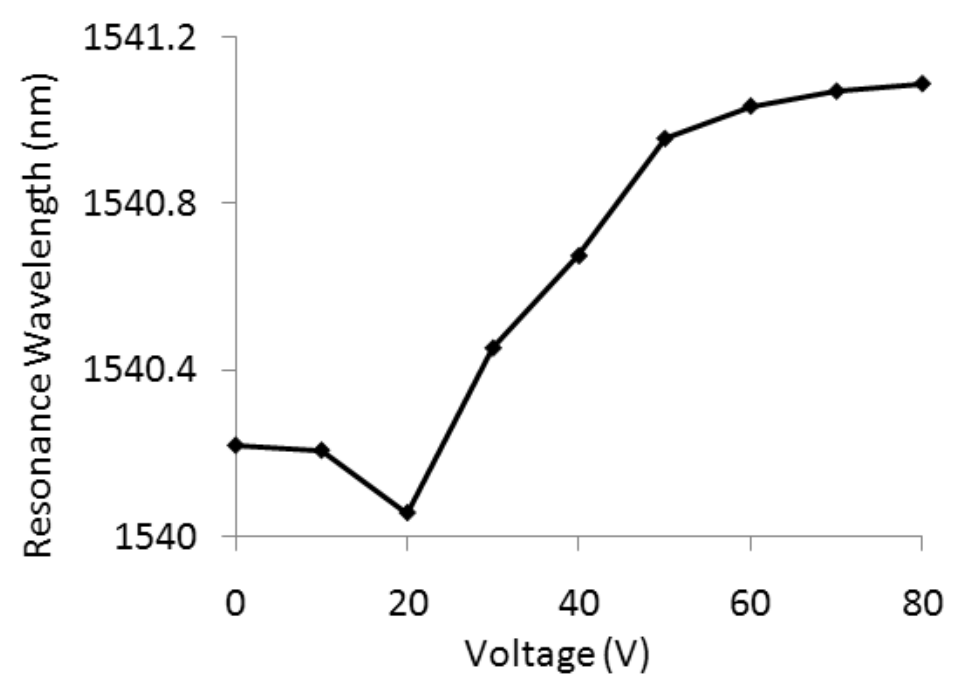

Fig. 6. When we trace the resonance wavelength for increasing voltages we find a curve with a threshold value and a saturation effect for high voltages. 

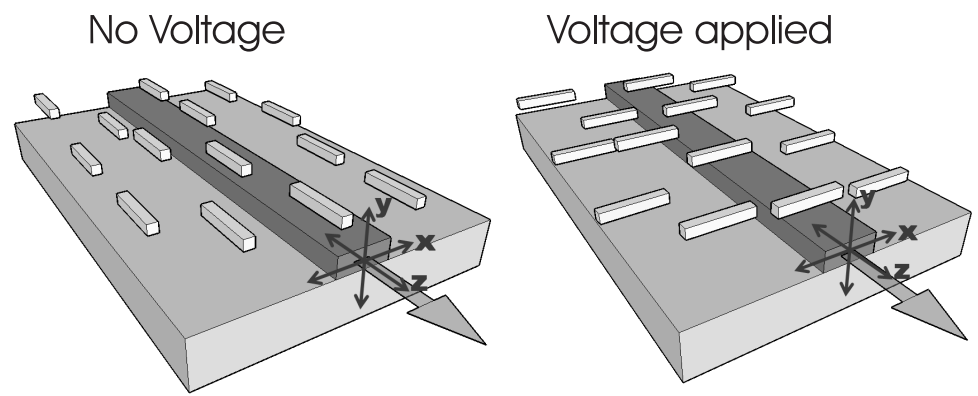

Fig. 7. When there is no voltage applied, the molecules align parallel to the waveguide. With the electric field present the molecules reorient in the plane parallel to the surface to align along the field lines perpendicular to the waveguide. 


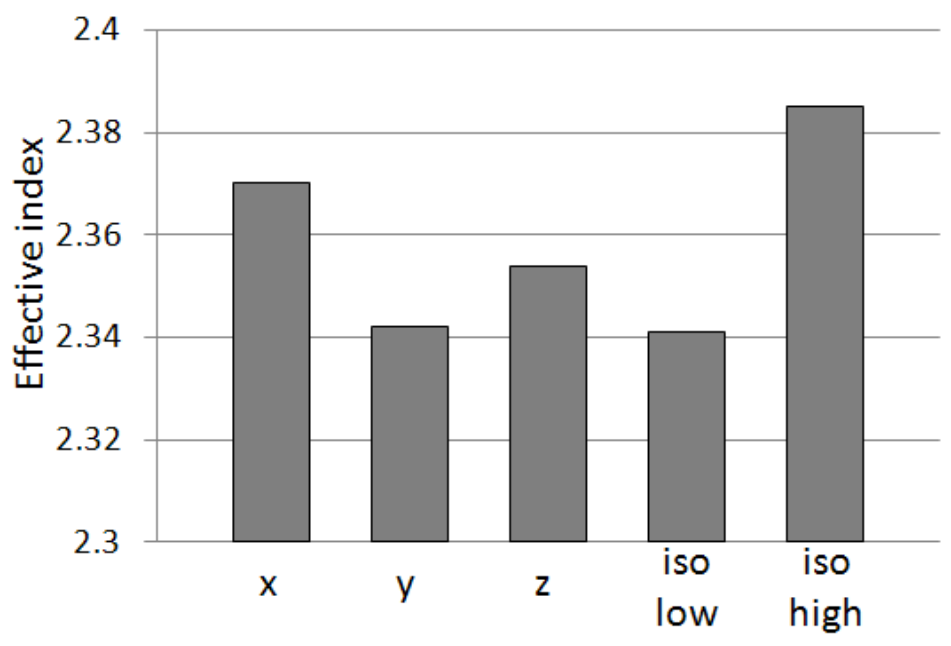

Fig. 8. We compare the effective index of the waveguide mode for different LC orientations. From left to right: LC oriented along x-, y-, and z-axis, uniform refractive index equal to the ordinary refractive index of the $\mathrm{LC}$ and uniform refractive index equal to the extra-ordinary refractive index of the LC. 


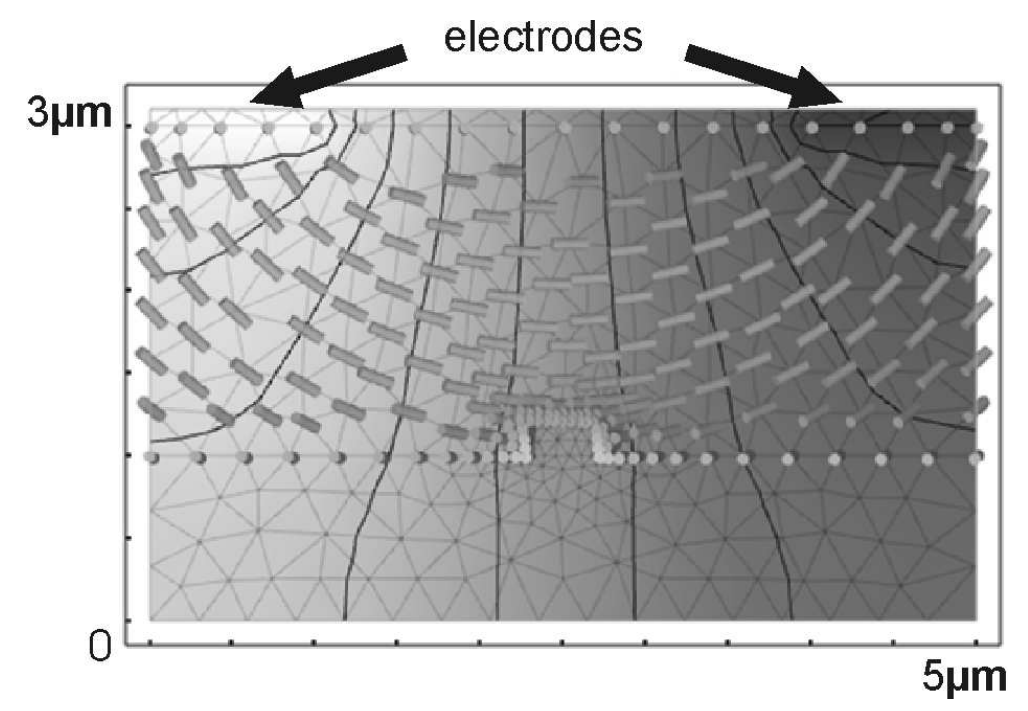

Fig. 9. We simulate the director orientation of the LC cladding on top of the waveguide in the presence of an electric field. The results are imported in the mode solver. 


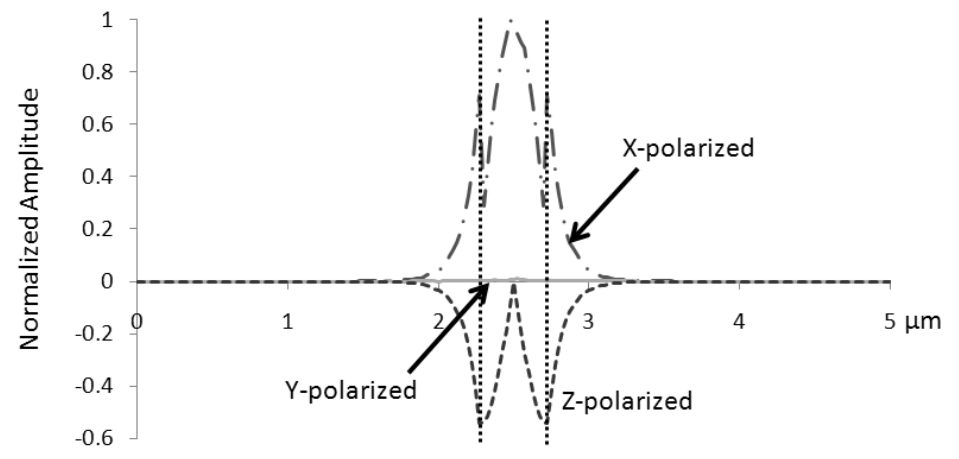

Fig. 10. With our mode solver we can calculate the $\mathrm{x}-$, $\mathrm{y}$ - and z-polarized fields of the light. We present here the normalized fields along a horizontal cut through the waveguide (see dotted line in Fig. 1). 


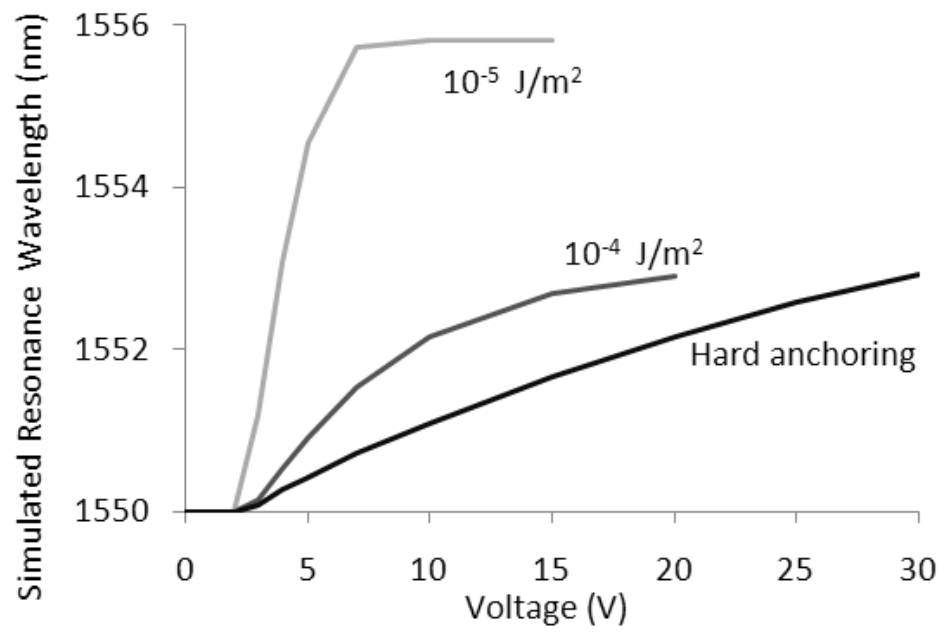

Fig. 11. Simulated resonance wavelength for increasing voltages. Three different values for the anchoring are used. For lower values of the anchoring on the surface the tuning range is wider and the saturation appears at lower voltages. 